Cahiers $d u$ MONDE RUSSE

\section{Cahiers du monde russe}

Russie - Empire russe - Union soviétique et États indépendants

$53 / 4 \mid 2012$

Varia

\title{
Rajnhard Nahtigal', Murmanskaja železnaja doroga 1915-1919 gg
}

Alexandre Sumpf

\section{OpenEdition}

\section{Journals}

Édition électronique

URL : http://journals.openedition.org/monderusse/7809

DOI : $10.4000 /$ monderusse.7809

ISSN : $1777-5388$

Éditeur

Éditions de l'EHESS

\section{Édition imprimée}

Date de publication : 15 décembre 2012

ISSN : $1252-6576$

Référence électronique

Alexandre Sumpf, « Rajnhard Nahtigal', Murmanskaja železnaja doroga 1915-1919 gg », Cahiers du monde russe [En ligne], 53/4 | 2012, mis en ligne le 02 décembre 2013, Consulté le 22 septembre 2020. URL : http://journals.openedition.org/monderusse/7809; DOI : https://doi.org/10.4000/monderusse. 7809

Ce document a été généré automatiquement le 22 septembre 2020.

(c) École des hautes études en sciences sociales 


\title{
Rajnhard Nahtigal', Murmanskaja železnaja doroga 1915-1919 gg
}

\author{
Alexandre Sumpf
}

\section{RÉFÉRENCE}

Rajnhard NAHTIGAL' [Reinhard Nachtigal], Murmanskaja železnaja doroga

1915-1919 gg. Voennaja neobhodimost' i èkonomičeskie soobraženija [La voie de chemin de fer de Mourmansk, 1915-1919. Nécessité de guerre et considérations économiques]. Sankt Peterburg : Nestor-Istorija, 2011, 320 p.

1 Cet ouvrage reprend et augmente les travaux antérieurs de l'auteur publiés en allemand. Reinhard Nachtigal a rencontré la ligne de chemin de fer joignant la capitale Petrograd au nouveau port de Mourmansk (alors Romanov-sur-Mourman), sur la mer Blanche, lors de son étude du sort des prisonniers de guerre allemands en Russie. Une partie de ses réflexions porte donc logiquement sur l'emploi de ces ressortissants étrangers à la construction de cette infrastructure. Cette analyse s'insère dans une approche économique, diplomatique et militaire plus globale, ce qui en fait l'intérêt. À travers cette étude de cas, on saisit un peu mieux les tensions contradictoires à l'œuvre en Russie au cœur du «continuum de la crise » (1914-1921) cher à Peter Holquist. Si le plan adopté est chronologique, la problématisation des chapitres est thématique - le labeur des prisonniers, les mesures de rétorsion allemandes, la complexe implication anglaise. Pour aider le lecteur, de passionnants diagrammes articulent la démonstration sur la répartition par nationalité et lieu de travail des prisonniers de guerre. En revanche, les illustrations photographiques sont très décevantes : leur choix paraît n'obéir à aucun principe puisque se mêlent clichés du xxi siècle sans intérêt (façades d'immeubles) et images d'époque, hélas jamais commentées en dépit de détails intrigants, et surtout jamais clairement référencées.

2 Comme souvent dans l'histoire de l'industrialisation russe, le rôle des étrangers et de l'étranger apparaît ici dans toute son ampleur. La chronique de l'installation du chemin 
de fer dans ce qui constituera plus tard la république autonome de Carélie est transnationale : la guerre ouvre le dossier, les alliés anglais jouent le rôle de conseillers techniques et d'aiguillons aux considérations stratégiques, les alliés américains les supplantent dans celui de fournisseur de matériel - et quelque 70000 prisonniers de guerre ont malgré eux, et en dépit du droit de la guerre, aidé 10000 ouvriers autochtones à réaliser cet ouvrage. Par rapport à Arhangel'sk, Mourmansk présente l'avantage de ne pas geler l'hiver; en revanche, l'accès à la mer Blanche est en partie contrôlé par les sous-marins allemands. L'intérêt stratégique du port, réel, ne doit donc pas être surévalué - les acteurs de l'époque en avaient pleinement conscience. Les pages consacrées à l'investissement britannique éclairent un pan souvent mal connu des relations entre alliés. On est frappé par l'absence d'expertise approfondie de la faisabilité et de l'intérêt concret de la voie, comme si l'important était surtout de poser un pied ferme à cette extrémité de l'Europe ouverte sur la Russie, mais aussi la Baltique. Les échecs répétés des entrepreneurs privés se lançant dans l'aventure, et l'attitude hésitante du gouvernement britannique, ont contribué autant que les contradictions russes à réduire un grand projet d'aménagement à un très modeste moyen de communication.

3 Techniquement, beaucoup laisse en effet à désirer. La voie finalement réalisée, à sens unique, pâtit de graves défauts de construction provoqués par la hâte, l'emploi de travailleurs peu qualifiés avec un turnover élevé, les conditions pédologiques et météorologiques. Les rapports cités par Nachtigal insistent lourdement sur l'impréparation, les mauvaises surprises réservées par un terrain constitué de marais et de permafrost ou l'insuffisance de matériel de qualité (rails, traverses). Comme souvent en Russie (puis en URSS), c'est la force humaine qui compense le très faible apport de force mécanique ou même animale, dans des conditions climatiques assez rudes. Le terrain impose sa conclusion impitoyable: au lieu d'une ligne construite selon les standards internationaux, irrigant tout le nord de l'empire, Russes et Alliés devront se contenter d'un mince fil temporaire, soumis aux glissements de terrain et aux inondations. Les ouvriers doivent sans cesse réparer la voie, les accidents sont légion, la vitesse de transport oscille entre 10 et $20 \mathrm{~km} / \mathrm{h}$. Dans ces conditions, que transporte-ton? L'auteur ne le précise guère, et ne permet donc pas de saisir le niveau de la participation de la ligne à l'effort de guerre allié ni de comparer l'activité de Mourmansk à celle d'Arhangel'sk.

La discussion autour de l'emploi des prisonniers de guerre pendant la guerre en Russie gagne ici de précieux arguments. Nachtigal prouve, documentation russe à l'appui, que les autorités militaires ont non seulement sciemment utilisé cette main d'œuvre forcément bon marché et forcée d'obtempérer, mais que l'on a organisé des transferts spéciaux depuis les camps de Sibérie à cet effet, et qu'une politique de ségrégation nationale a été mise en œuvre. Le tournant de 1915 dans ce domaine s'observe donc en Russie à l'instar des autres nations en guerre. Les conditions de travail, dont la description rappelle parfois un rapport sur le Goulag des années 1930, découlent en réalité de la pauvreté des moyens engagés et de la médiocre attention à cette question, récurrente en Russie. Le sort fait ici aux prisonniers de guerre n'est à bien des égards pas bien pire que dans le reste de l'Europe - même si la mortalité y est importante. Cette dernière découle non de mauvais traitements, mais des conditions extrêmes et du travail harassant. La répartition des détenus le long du tracé ne permet pas de prouver la pratique d'affectations punitives pour des groupes ou des individus. Il aurait été intéressant, cela dit, de disposer de données autorisant la comparaison avec les 10000 
travailleurs libres employés aux côtés des détenus, et de témoignages des acteurs permettant l'appréciation de la situation au quotidien.

5 Les Allemands du Reich (23\%) ou Autrichiens (25\%) représentent un peu moins de la moitié des effectifs, soit plus que les Hongrois (38\%) et les Slaves (environ 10\%). Il y avait de fait bien moins de prisonniers de l'armée allemande que de l'armée austrohongroise internés en Russie : si un quart des travailleurs forcés à Mourmansk sont des Allemands du Reich, cela n'excède pas leur proportion. C'est au sein de l'armée austrohongroise que les autorités militaires russes ont procédé à un tri. La sélection favorable aux Slaves appliquée dans la plupart des camps précède toutefois la décision de l'envoi en Carélie. À la même époque, si l'on suit Oksana Nagornaja dans Drugoj voennyj opyt (2010), les autorités allemandes agissent de même pour essayer, sans grand succès, de créer une scission entre Russes d'un côté, et Ukrainiens ou musulmans de l'autre. Les protestations allemandes n'ont de ce fait qu'une portée limitée, quel que soit l'argument avancé. L'emploi des prisonniers de guerre sur la ligne de chemin de fer contrevient aux conventions de La Haye, que les belligérants respectent quand cela les arrange (rarement, donc). L'appel aux rétorsions envers les combattants russes internés en Allemagne apparaît comme une intimidation de faible portée. S'il y a un durcissement du régime, comme l'a démontré Heither Jones, c'est par suite de la politique de séparation des officiers et des soldats du rang et de ségrégation nationale, et du fait qu'en 1916, le pays commence à souffrir intensément sur le plan alimentaire. Outre les visites de sœurs de charité de la Croix-Rouge ou les missives échangées entre Nicolas II et Guillaume II, un autre champ de lutte, que l'auteur explore peu, est celui de la propagande autour des "atrocités de l'ennemi». La Commission extraordinaire d'enquête du sénateur Krivcov multiplie les brochures sur les mauvais traitements des prisonniers de guerre russe ; qu'en est-il en Allemagne, et comment répond-on dans le Reich à ces témoignages ? Le cas précis de la ligne de Mourmansk ne paraît pas avoir provoqué d'effort particulier de contre-propagande.

Un dernier point, abordé à la marge ici, méritait sans doute une réflexion un peu plus poussée. À le lire, on sent que cette opération a tout d'un projet colonial, que ce soit du point de vue de l'impérialisme britannique - qui se concrétisera brièvement en 1919 dans le cadre de l'intervention étrangère dans la guerre civile russe - ou du pouvoir central russe. Quels étaient les rapports entre ce dernier et les autorités locales? Comment était perçue cette frontière au sens géopolitique et économique: représentait-elle un nouvel horizon national qui expliquerait le rôle à l'époque soviétique de ce Grand Nord, étudié par Yuri Slezkine et Samuel Baron? On le voit, la riche étude de cas proposée par Nachtigal ouvre l'accès à de nombreux enjeux historiographiques, sur une période de l'histoire russe pour laquelle tant reste à défricher. 